\title{
ON LEFT STRONG RADICALS OF NEAR-RINGS
}

\author{
by T. ANDERSON, K. KAARLI and R. WIEGANDT*
}

(Received 12th January 1987)

\section{Introduction}

In this paper we shall deal with radicals $\gamma$ of near-rings such that for every near-ring $N$ its radical $\gamma(N)$ contains all left ideals (left invariant subgroups, respectively) $I$ of $N$ with $I \in \gamma$. At first, examples of such radicals will be given. Then we shall prove that these radicals are hypersolvable and have hereditary semisimple classes.

\section{Preliminaries}

A near-ring will always mean a left near-ring, that is, a near-ring satisfying

$$
x(y+z)=x y+x z .
$$

With the exception of Section 3 we shall consider only 0-symmetric near-rings, that is, near-rings satisfying

$$
0 x=x \text {. }
$$

If $I$ is an ideal (left ideal or normal subgroup) of a near-ring $N$, then we shall write $I \triangleleft N, I \succ_{l} N$ or $I-N$, respectively. A subgroup $K$ of a near-ring $N$ is called a left invariant subgroup of $N$, if $N K \subseteq K$; this fact will be denoted by $K{ }_{t} N$. A left ideal is always left invariant, but a left invariant subgroup need not be a normal subgroup and hence it need not be a left ideal. For an $N$-group $G$ and subset $H \subseteq G$ we shall often consider the Noetherian quotient

$$
(H: G)_{N}=\{n \in N: G n \subseteq H\} .
$$

Whenever we shall speak of a class $\mathbb{C}$ of near-rings, we suppose that $\mathfrak{C}$ is closed under isomorphisms and contains the near-ring 0 . A class $\mathfrak{A}$ is called a universal class, if $\mathfrak{A}$ is homorphically closed and hereditary (that is, $I \triangleleft N \in \mathfrak{U}$ imply $I \in \mathfrak{Q}$ ).

We shall say that a class $\mathbb{C}$ of near-rings is regular (left invariantly regular), if for any $N \in \mathbb{C}$ and $0 \neq I \sqcup N(0 \neq I \sqcup, N$, respectively) there exists a homomorphic image $K$ of $I$ such that $0 \neq K \in \mathfrak{C}$. A class $\mathbb{C}$ is said to be subdirectly closed, if every subdirect sum of

\footnotetext{
* Research partially supported by Hungarian National Foundation for Scientific Research grant No. 1813.
} 
near-rings from $\mathfrak{C}$ is again in $\mathfrak{C}$. If for any $I \triangleleft N, I \in \mathbb{C}$ and $N / I \in \mathbb{C}$ imply $N \in \mathfrak{C}$, then we shall say that the class $\mathbb{C}$ is closed under extensions.

In any universal class $\mathfrak{A}$ of near-rings the Kurosh-Amitsur radical theory can be developed. Let us recall that a subclass $\gamma$ of $\mathfrak{U}$ is called a radical class (or briefly a radical), if $\gamma$ is homomorphically closed, $\gamma$ is closed under extensions and for every nearring $N$

$$
\gamma(N)=\Sigma(I \triangleleft N: I \in \gamma) \in \gamma
$$

The ideal $\gamma(N)$ is called the $\gamma$-radical of the near-ring $N$.

To any radical class $\gamma$ there belongs the semisimple class

$$
\mathscr{S} \gamma=\{N \in \mathfrak{A}: \gamma(N)=0\},
$$

any any regular class $\sigma$ determines the upper radical class

$$
\gamma=\mathscr{U} \sigma=\{N \in \mathfrak{U}: N \text { has only } 0 \text { homorphic image in } \sigma\} .
$$

Every semisimple class is regular, and $\gamma=\mathscr{U} \mathscr{S}_{\gamma}, \sigma=\mathscr{S} \mathscr{U} \sigma$ are valid for every radical class $\gamma$ and semisimple class $\sigma$. If $\gamma$ is a radical, then for every near-ring $N$

$$
\gamma(N)=\cap(I \triangleleft N: N / I \in \mathscr{S} \gamma)
$$

holds.

We say that a radical $\gamma$ is left strong (left invariantly strong), if $L \in \gamma$ and $L \triangleleft_{1} N$ $\left(L-{ }_{l} N\right.$, respectively) imply $L \subseteq \gamma(N)$. Obviously every left invariantly strong radical is also left strong. Left strong radicals of near-rings (more precisely, right strong radicals of right near-rings) were discussed in the recent paper [8] of Mlitz and Oswald. The examples will show that some of the important radicals are left invariantly strong, justifying the investigations of such radicals. As is well-known in the universal class of all associative rings left invariantly strong radicals are left strong radicals and the Baer, the Levitzki and the Jacobson radicals are examples for such radicals, but the BrownMcCoy radical is not.

For the fundamentals of radical theory and for more details on near-rings we refer to [12] and [9], respectively.

\section{Examples for left invariantly strong radicals}

In this section we shall work in the universal class of all 0-symmetric near-rings.

An $N$-group $G$ is said to be of type 2 , if $G N \neq 0$ and $G$ has no non-trivial $N$-subgroups. The $J_{2}$-radical $J_{2}(N)$ of a near-ring $N$ is defined as the intersection

$$
J_{2}(N)=\cap\left\{(0: G)_{N}: G \text { is of type } 2\right\} .
$$

(If the intersection is taken over the empty set, then $J_{2}(N)=N$.) Kaarli [6] has proved that $J_{2}$ is a hereditary Kurosh-Amitsur radical with hereditary semisimple class. 
Example 1. $J_{2}$ is a left invariantly strong radical.

In order to see this, we have to show that any $J_{2}$-semisimple near-ring $N$ does not contain non-zero left invariant subgroups in $J_{2}$. Let $N$ be a near-ring such that $J_{2}(N)=0$, and let $K \neq 0$ be a left invariant subgroup of $N$. $K \notin J_{2}$ will be proved, if we show that there exists a $K$-group of type 2. Since $J_{2}(N)=0$ and $K \neq 0$, there exists an $N$-group $G_{N}$ of type 2 such that $G_{N} K \neq 0$. Hence there exists an element $g \in G_{N}$ such that $H=g K \neq 0$. We claim that $H$ is a $K$-group of type 2. For an arbitrary element $h \in H$ either $h N=0$ or $h N=G_{N}$. If $h N=G_{N}$, then $g=h n$ for some $n \in N$, and

$$
H=g K=h n K \subseteq h K,
$$

as $K$ is left invariant in $N$. Thus we have

$$
H=h K \subseteq(g K) K \subseteq g K=H
$$

which shows that $H$ is the union

$$
H=\{h \in H: h N=0\} \cup\{h \in H: h K=H\}
$$

Hence for any $K$-subgroup $F \neq 0$ of $H$ either $F=f K=H$ for some $f \in H$, or $F K=0$. The last case is not possible, because $G_{N}$ is of type 2 . Thus $H$ is a $K$-group of type 2.

An $N$-group $G$ is said to be of type 3 , if $G$ is of type 2 and if for any $g_{1}, g_{2} \in G$ the validity of $g_{1} n=g_{2} n$ for all $n \in N$ implies $g_{1}=g_{2}$. The $J_{3}$-radical of a near-ring $N$ is the intersection

$$
J_{3}(N)=\cap\left\{(0: G)_{N}: G \text { is of type } 3\right\}
$$

Holcombe and Walker [4] have shown that $J_{3}$ is a Kurosh-Amitsur radical, and Holcombe [3] has proved that $J_{3}$ is hereditary with hereditary semisimple class.

Example 2. $J_{3}$ is a left invariantly strong radical.

We shall show that a near-ring $N$ such that $J_{3}(N)=0$, has no non-zero left invariant subgroup in $J_{3}$. Let $K \neq 0$ be a left invariant subgroup of $N$. Since $J_{3}(N)=0$ and $K \neq 0$, there exists an $N$-group $G_{N}$ of type 3 such that $G_{N} K \neq 0$. Since an $N$-group of type 3 is also of type 2, there exists an element $g \in G_{N}$ such that $H=g K \neq 0$ is a $K$-group of type 2 (see the proof in Example 1). We show that $H$ is of type 3. Let $g_{1}$ and $g_{2}$ be elements of $H$ such that $g_{1} k=g_{2} k$ for all $k \in K$. If $g_{1}=g_{2}=0$, then we are done. Assume that $g_{1} \neq 0$. Since $G_{N}$ is of type 3, we have $g_{1} N=G_{N}$ and, as in Example 1, also $g_{2} K=H$. Now we distinguish two cases.

(i) $\left(0: g_{1}\right)_{N}=\left(0: g_{2}\right)_{N}$. In this case we have an $N$-automorphism $\alpha$ of $G_{N}$ defined by $\alpha\left(g_{1} n\right)=g_{2} n, n \in N$. Since $g_{1} k=g_{2} k$ for every $k \in K, H$ is contained in

$$
F=\{x \in G: \alpha(x)=x\}
$$


$F$ is obviously an $N$-subgroup of $G$, so $F=G$ and $\alpha=1$, because $G_{N}$ is of type 2 . Hence $g_{1} n=g_{2} n$ for every $n \in N$ implying $g_{1}=g_{2}$ for $G_{L}$ is of type 3 .

(ii) $\left(0: g_{1}\right)_{N} \neq\left(0: g_{2}\right)_{N}$. In view of Ramakotaiah [11, Theorem 4], there exists an $n \in N$ such that $g_{1} n=g_{1}$ and $g_{2} n=0$. Therefore taking into account that $K$ is a left invariant subgroup of $N$, we get $g_{1} k=g_{1} n k=g_{2} n k=0$ for all $k \in K$, contradicting $g_{1} K=H$.

In a similar way one can define many other left invariantly strong radicals. Let us consider a given cardinal $\tau$ and the class

$$
\mathfrak{M}(\tau)=\{G: G \text { is an } N \text {-group of type } 2 \text { and }|G| \leqq \tau\},
$$

and to every near-ring $N$ let us assign the ideal

$$
J_{(2, \tau)}(N)=\cap\left\{(0: G)_{N}: G \in \mathfrak{M}(\tau)\right\}
$$

Similarly to the case of $J_{2}$ one can prove that this assignment defines a Kurosh-Amitsur radical and, as above, one can see that this radical is left invariantly strong. Considering $N$-groups of type 3 instead of $N$-groups of type 2, one gets left strong radicals $J_{(3, \tau)}$.

Example 3. The nil radical $\mathscr{N}$ consisting of all nil near-rings is obviously hereditary and it has non-hereditary semisimple class, as it follows easily from [7, Example 5.4]. The nil radical $\mathcal{N}$ is not left (invariantly) strong. Let $G$ be a finite abelian group and $H \neq 0$ a proper subgroup of $G$. Denote by $M_{0}(G)$ the near-ring of all mappings $f: G \rightarrow G$ such that $0 f=0$, and let

$$
N=\left\{n \in M_{0}(G): H n=0\right\},
$$

and $L=(H: G)_{N}$. A straightforward computation shows that $G_{N}$ is of type 1 (that is, for all $g \in G$ either $g N=G$ or $g N=0$ and $G$ is a simple $N$-group. It has been shown in [10, Corollary 2.6] that a near-ring $N$ having a faithful $N$-group $G_{N}$ of type 1 is necessarily simple. Obviously $N^{2} \neq 0$, and so $N$ is nil semisimple. Further, $L$ is clearly a left ideal of $N$ and $L^{2}=0$. Hence the radical $\mathcal{N}$ is not left strong. Let us notice that in the universal class of all associative rings the question whether the nil radical is left strong, is the famous and still open problem of Köthe.

Next, let $\mathscr{M}$ denote any class of left invariantly simple near-rings (that is, near-rings having only trivial left invariant subgroups) with identity, for instance, a class of nearfields. By [1, Theorem 3] the subdirect closure

$$
\mathscr{M}=\{N: N \text { is a subdirect sum of near-rings from } \mathscr{M}\}
$$

is just the semisimple class of the upper radical

$$
\mathscr{U} \mathscr{M}=\{N: N \text { has only } 0 \text { homorphic image in } \mathscr{M}\} .
$$

Example 4. UU $\mathscr{M}$ is a left invariantly strong radical. 
By [13, Corollary 4] it suffices to show that $\mathscr{H}$ is left invariantly regular. Let us consider a non-zero left invariant subgroup $L$ of a near-ring $N \in \mathscr{M}$. Since $N \in \bar{M}$, there exists an ideal $I$ of $N$ such that $N / I \in \mathscr{M}$ and $L \nsubseteq I$. Hence we have

$$
0 \neq L /(L \cap I) \simeq(L+I) / I \hookrightarrow_{l} N / I \in \mathscr{M} .
$$

Taking into consideration that $N / I$ is left invariantly simple, we get $(L+I) / I=N / I$, and so $L$ has a non-zero homorphic image in $\mathscr{M}$.

Let us remind the reader that in the universal class of all associative rings the BrownMcCoy radical is not left strong, hence it is not left strong in the universal class of all near-rings either.

One can easily construct examples of left strong radicals which are not left invariantly strong. Let us consider, for instance, the near-ring $N_{5}$ with zero-multiplication built on the additively written alternating group $A_{5}$ of permutations of 5 elements. $A_{5}$ is simple and is of order 60 , so it has a 2-Sylow subgroup $G \neq 0$ which is not normal in $A_{5}$. Let $\zeta_{2}$ denote the lower left strong radical class generated by all near-rings with zeromultiplication and having additive order $2^{n}, n=1,2, \ldots$ in the universal class of all nearrings. Obviously $N_{5} \notin \zeta_{2}$ but $G \in \zeta_{2}$. Hence $\zeta_{2}$ is not left invariantly strong. Nevertheless, we do not know whether among the so far discussed natural near-ring radicals there is a left strong radical which is not left invariantly strong.

\section{The hypersolvability of left strong radicals}

In this section we shall work in a universal class $\mathfrak{Q}$ satisfying the following additional requirement:

(*) if $A, B \in \mathfrak{U}$ and $C$ is a near-ring such that $C^{+} \simeq A^{+} \oplus B^{+}$, then also $C \in \mathfrak{A}$,

where $N^{+}$stands for the additive group of $N$. Obviously the universal classes of all near-rings and all finite near-rings do satisfy condition (*). Moreover, any universal class of near-rings with additive groups belonging to a given variety (for example, abelian groups) satisfy condition (*).

A radical $\gamma$ is called hypersolvable, if $\gamma$ contains all near-rings of the universal class with zero multiplication. Let us observe that all examples of left invariantly strong radicals given in Section 2 are hypersolvable. In fact, we have the following more general result.

Theorem 1. Let $\mathfrak{A}$ be a universal class of (not necessarily 0-symmetric) near-rings which satisfies condition (*). If $\gamma \neq 0$ is a left strong radical class in $\mathfrak{A}$, then $\gamma$ is hypersolvable.

Proof. Suppose that $\gamma$ is not hypersolvable. Then there exists a near-ring $S \in \mathscr{S} \gamma$ such that $S \neq 0$ and $S^{2}=0$. Let us take an arbitrary near-ring $N \in \gamma, N \neq 0$, and define a nearring $\mathscr{K}(N, S)$ on the cartesian product $N \times S \times S$ by the componentwise addition and by 
the multiplication

$$
\left(n_{1}, s_{1}, t_{1}\right)\left(n_{2}, s_{2}, t_{2}\right)=\left\{\begin{array}{l}
\left(n_{1} n_{2}, t_{2}, 0\right) \text { if } n_{1} \neq 0 \text { and } t_{1} \neq 0 \\
\left(n_{1} n_{2}, 0,0\right) \text { if } n_{1}=0 \text { or } t_{1}=0
\end{array}\right.
$$

It is easy to check that $\mathscr{K}(N, S)$ is really a near-ring. Moreover, by condition (*) the near-ring $\mathscr{K}(N, S)$ is a member of the universal class $\mathfrak{U}$, and $\mathscr{K}(N, S)$ is 0 -symmetric whenever $N$ is so. Let us consider the subsets

$$
X=\{(n, s, 0): n \in N, s \in S\}
$$

and

$$
Y=\{(n, 0,0): n \in N\}
$$

of $\mathscr{K}(N, S)$. It is easy to see that

$$
X \sqsupset \mathscr{K}(N, S), \quad \mathscr{K}(N, S) / X \simeq S
$$

and

$$
N \simeq Y \triangleleft X, \quad X / Y \simeq S
$$

Hence by $S \in \mathscr{S}_{\gamma}$ we get $\gamma(\mathscr{K}(N, S)) \subseteq X$, and therefore also $\gamma(\mathscr{K}(N, S)) \subseteq \gamma(X)$ holds. Since $X / Y \simeq S \in \mathscr{S}_{\gamma}$, we have $\gamma(X) \subseteq Y$. These together yield $\gamma(\mathscr{K}(N, S)) \subseteq Y$. Since

$$
\left(n_{1}, s_{1}, t_{1}\right)\left(n_{2}, 0,0\right)=\left(n_{1} n_{2}, 0,0\right) \in Y,
$$

$Y$ is a left invariant subgroup, in fact, also a left ideal of $\mathscr{K}(N, S)$. Since $\gamma$ is left strong and $Y \simeq N \in \gamma$, we conclude $Y \subseteq \gamma(\mathscr{K}(N, S))$. Thus $Y=\gamma(\mathscr{K}(N, S)) \Delta \mathscr{K}(N, S)$ holds. We have, however,

$$
\begin{gathered}
{\left[\left(0, s_{1}, t_{1}\right)+(k, 0,0)\right]\left(n_{2}, s_{2}, t_{2}\right)-\left(0, s_{1}, t_{1}\right)\left(n_{2}, s_{2}, t_{2}\right)} \\
\quad=\left(k n_{2}, t_{2}, 0\right)-\left(0 n_{2}, 0,0\right)=\left(k n_{2}-0 n_{2}, t_{2}, 0\right) \notin Y
\end{gathered}
$$

whenever $t_{2} \neq 0$. Hence $Y$ is not a right ideal of $\mathscr{K}(N, S)$, contradicting $Y=\gamma(\mathscr{K}(N, S))$. Thus $\gamma$ must be hypersolvable.

Let us emphasize that Theorem 1 is valid also for any universal class of 0-symmetric near-rings satisfying condition (*). The assertion, however, fails to be true for associative rings as $\mathscr{K}(N, S)$ is not a ring even if $N$ is a ring. 


\section{The heredity of semisimple classes of left invariantly strong radicals}

Having assumed condition (*) for the universal class Mlitz and Oswald ([8, Corollary 2.6]) proved recently that the semisimple class of a left strong radical is hereditary, provided that the semisimple class consists of 0 -symmetric near-rings. In this section we shall prove without imposing condition (*) that every left invariantly strong radical has a hereditary semisimple class in any universal class of 0-symmetric near-rings. Any universal class of near-rings which contains the ring of integers but does not contain the zero-ring on the infinite cyclic group does not satisfy condition (*). We do not know, however, whether in such a universal class a left invariantly strong radical with hereditary semisimple class has to be hypersolvable. Our proof does not work for left strong radicals.

First we prove the following.

Proposition 1. Let $M \triangleleft I \triangleleft N$. Then for any element $n \in N$ the map $f: M \rightarrow(n M+M) / M$ defined by $f(m)=n m+M, m \in M$, is a surjective homomorphism and $(n M+M) / M$ is a near-ring with zero-multiplication.

Proof. $f$ is clearly an additive homomorphism which is surjective. Since $N$ is 0 -symmetric, we have

$$
(N M)^{2}=N(M N) M \subseteq N I M \subseteq I M \subseteq M .
$$

Hence

$$
f\left(m_{1}\right) f\left(m_{2}\right) \in(N M+M)(N M+M) \subseteq(N M)^{2}+M \subseteq M
$$

and

$$
f\left(m_{1} m_{2}\right) \subseteq n M M+M \subseteq I M+M \subseteq M .
$$

Thus $f$ is a homomorphism and $(n M+M) / M$ is a near-ring with zero-multiplication.

Theorem 2. If $\gamma$ is a left invariantly strong radical (in a universal class of 0-symmetric near rings), then its semisimple class $\mathscr{S}_{\gamma}$ is hereditary.

Proof. Let $N$ be a near-ring such that $N \in \mathscr{S} \gamma$. For any $I \triangleleft N$ we put $M=\gamma(I)$. By Proposition 1 we can map $M$ homomorphically onto $(n M+M) / M$ for any $n \in N$. Since In $M \subseteq I M \subseteq M$, the factor near-ring $(n M+M) / M$ is a left invariant subgroup of $I / M$. Since $(n M+M) / M$, as a homomorphic image of the $\gamma$-radical near-ring $M$, is in $\gamma$, and $\gamma$ is left invariantly strong, we have

$$
(n M+M) / M \subseteq \gamma(I / M)=0 .
$$

This implies $n M \subseteq M$ for all $n \in N$, proving that $M$ is a left invariant subgroup of $N$. Since $M \in \gamma$ and $\gamma$ is left invariantly strong, it follows that $M \subseteq \gamma(N)=0$, that is, $I \in \mathscr{S}_{\gamma}$. 
Betsch and Kaarli [2] have proved (using condition (*)) that if a semisimple class of (not necessarily 0 -symmetric) near-rings is hereditary, then its upper radical is hypersolvable. Combining this with our Theorem 2 we recover the assertion of our Theorem 1 restricted to left invariantly strong radicals in universal classes of 0 -symmetric near-rings.

Since in the universal class of all 0 -symmetric near-rings the nil radical has nonhereditary semisimple class (cf. [7, Example 5.4]) by Mlitz and Oswald [8, Corollary 2.6] (or by our Theorem 2 for left invariantly strong radicals) we have got another proof for the assertion of Example 3.

\section{The heredity of semisimple classes of left strong radicals}

In this section we shall prove a version of the previously quoted [8, Corollary 2.6 ] of Mlitz and Oswald: the assumptions will be different, meanwhile the conclusion remains the same. Thus not so much the result, but the method used in the proof, seems to be of interest. The universal class $\mathfrak{A}$ we shall work with consists of 0 -symmetric near-rings and need not satisfy condition (*), but satisfies the following requirement:

(**) for every $n \in N \in \mathfrak{A}$ and $M \hookrightarrow N$ also $n M \hookrightarrow N$ holds.

Conditions (**) means that left multiplication is compatible with addition in the following sense: left multiplication maps normal subgroups to normal subgroups. Condition (**) is satisfied, for instance, in the universal class of abelian near-rings and in that of near-rings with Hamiltonian additive groups. The universal class of all abelian near-rings and that of all finite abelian near-rings satisfy beside (**) also (*), but it may happen that only (**) is satisfied as, for instance, in the universal class of all abelian near-rings which contain the ring of integers, but does not contain the zero-ring on the infinite cyclic group.

We shall make use of the following three propositions.

Proposition 2. Let $\gamma$ be a left strong radical in a universal class $\mathfrak{U}$ of near-rings satisfying condition (**). If $I \triangleleft N, I^{2}=0$ and $\gamma(N)=0$, then $\gamma(I)=0$.

Proof. Put $M=\gamma(I)$. For any additive automorphism $\alpha$ of $I$ we have $\alpha M-\alpha I=I$. Since $M \in \gamma$ and $M^{2} \subseteq I^{2}=0$, we have $\alpha M \in \gamma$ and $\alpha M \triangleleft I$. Hence $\alpha M \subseteq M$ by the definition of $M$. In particular, for every $n \in N$ we have $n+M-n \subseteq M$ and hence $M-N$.

Next, let us consider an arbitrary element $n \in N$. By condition (**) we have $n M-N$ and so $n M \triangleleft I$, too. Since $I^{2}=0$, we have $n M \triangleleft I$. By $M^{2}=0, n M$ is a homomorphic image of $M \in \gamma$, and therefore also $n M \in \gamma$ holds for all $n \in N$. Hence $n M \subseteq \gamma(I)=M$ and by $M \triangleleft N$ we have also $M \triangleleft_{t} N$. Since $\gamma$ is left strong, we get $M \subseteq \gamma(N)=0$.

Proposition 3. If $M \triangleleft I \triangleleft N, y \in M$ and $x, n \in N$, then there exists an element $u \in(M: I)_{I}$ such that

$$
n+x y-n=x u
$$


Proof. Let $K$ denote the normal subgroup of $N$ generated by the element $y$. Then the elements of $K$ are of the form $u=\Sigma u_{a}$ where $u_{\alpha}=n_{\alpha}+k_{a} y-n_{a}$ with an integer $k_{\alpha}$ and an element $n_{a} \in N$. Using condition (**) we have $x K-N$, and so $n+x y-n \in x K$. Hence we get the identity

$$
n+x y-n=x u .
$$

If $y \in M$, then for any element $i \in I$ by the 0 -symmetry of $N$ we have

$$
i u=i \Sigma\left(n_{\alpha}+k_{\alpha} y-n_{\alpha}\right)=\Sigma\left(i n_{\alpha}+i k_{\alpha} y-i n_{\alpha}\right) \in M
$$

proving $u \in(M: I)_{I}$.

Proposition 4. If $M \triangleleft I \triangleleft N$, then for every element $n \in N$ the map $g: M \rightarrow(n+M-$ $n+M) / M$ defined by $g(m)=n+m-n+M, m \in M$, is a surjective homomorphism, and $(n+M-n+M) / M$ is a near-ring with zero-multiplication.

Proof. $g$ is obviously a surjective additive homomorphism, further also $g\left(m_{1}\right) g\left(m_{2}\right)=0$ holds, as $g(m) \in(M: I)_{I} / M$ and $(M: I)_{I}^{2} \subseteq I(M: I)_{I} \subseteq M$. It remains to show that $g\left(m_{1} \cdot m_{2}\right)=0$, that is, $n+m_{1} m_{2}-n \in M$. By Proposition 3, we have

$$
n+m_{1} m_{2}-n=m_{1} u
$$

where $u \in(M: I)_{I}$. Since by $[9,1.42$ Proposition $]$ we have $(M: I)_{I} \triangleleft I$, it follows

$$
n+m_{1} m_{2}-n \in m_{1}(M: I)_{I} \subseteq M(M: I)_{I} \subseteq M .
$$

Thus $g\left(m_{1} \cdot m_{2}\right)=0$ is true.

Theorem 3. Let $\mathfrak{A}$ be a universal class of 0-symmetric near-rings satisfying condition (**). If $\gamma$ is a left strong radical, then its semisimple class $\mathscr{S}_{\gamma}$ is hereditary.

Proof. It suffices to show that $I \triangleleft N$ implies $\gamma(I) \subseteq \gamma(N)$. Let $M=\gamma(I)$ and $P=(M: I)_{I}$. In view of $\left[9,1.42\right.$ Proposition] we have $P \triangleleft I$. Since $(P / M)^{2}=0$, Proposition 2 is applicable for $P / M \triangle I / M$ and for $\gamma(I / M)=0$, therefore also $\gamma(P / M)=0$ holds.

We claim that $M \rightarrow N$. Indeed, let $n \in N$ and consider $n+M-n$. Note that $n+M-n \subseteq P$, because $N$ is 0 -symmetric and so $I n \subseteq I$. Since for any $i \in I$ we have

$$
i+n+M-n-i=n+i^{*}+M-i^{*}-n \subseteq n+M-n,
$$

where $i^{*}=-n+i+n \in I$, we conclude $n+M-n-I$, and also

$$
n+M-n+M-P \text {. }
$$

Since $(P / M)^{2}=0$, it follows

$$
(n+M-n+M) / M \triangleleft P / M .
$$


By Proposition 4 the map $g(m)=n+m-n+M, m \in M$, is a homomorphism of $M$ onto $(n+M-n+M) / M$, and hence by $M \in \gamma$ also $(n+M-n+M) / M \in \gamma$ holds. Thus by $\gamma(P / M)=0$ we get $(n+M-n+M) / M=0$, that is, $n+M-n \subseteq M$ for all $n \in N$ proving $M-N$.

Next, consider any element $k \in N$. Condition $(* *)$ implies $k M-N$. Further, note that from the definition of $P$ it follows $k M \subseteq P$. Thus $(k M+M) / M \rightarrow P / M$ and by $(P / M)^{2}=0$ also $(k M+M) / M \triangleleft P / M$ holds. Now we can map $M$ homomorphically onto $(k M+$ $M) / M$ by $f(m)=k m+M, m \in M$, in view of Proposition 1. Since $\gamma(P / M)=0$, it follows $f(M)=0$, that is, $k M \subseteq M$ for all $k \in N$. Hence $M{ }_{l} N$. Taking into consideration that $M \in \gamma$ and that $\gamma$ is left strong, we get $\gamma(I)=M \subseteq \gamma(N)$.

\section{REFERENCES}

1. T. Anderson, K. KaArli and R. Wiegandt, Radical and subdirect decompositions, Comm. Algebra 13 (1985), 479-494.

2. G. Betsch and K. KaARLI, Supernilpotent radicals and hereditariness of semisimple classes of near-rings, Coll. Math. Soc. J. Bolyai 38 (Radical Theory, Eger, 1982, North-Holland, 1985) 47-58.

3. М. Ноцсомве, A hereditary radical for near-rings, Studia Sci. Math. Hungar. 17 (1982), 453456.

4. M. Holcombe and R. Walker, Radicals in categories, Proc. Edinburgh Math. Soc. 21 (1978/79), 111-128.

5. K. KaARLI, Minimal ideals in near-rings, Tartu Riikl. Ul. Toimetised 366 (1975), 105-142 (in Russian).

6. K. KaArl, Radicals in near-rings, Tartu Riikl. Ul. Toimetised 390 (1976), 134-171 (in Russian).

7. K. KaARLI, Classification of irreducible $R$-groups over a semiprimary near-ring, Tartu Riikl. Ul. Toimetised 556 (1981), 47-63 (in Russian).

8. R. Muttz and A. Oswald, Hypersolvable and supernilpotent radicals, Studia Sci. Math. Hungar. 24 (1989), to appear.

9. G. Pilz, Near-rings (North-Holland, 1977).

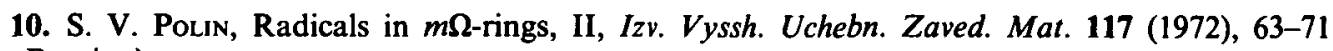
(in Russian).

11. D. Ramaxotaiah, Structure of 1-primitive near-rings, Math. Z. 110 (1969), 15-26.

12. R. Wiegand, Radical and Semisimple Classes of Rings (Queen's Papers in Pure and Appl. Math. 37, Kingston, Ontario, 1974).

13. R. Wiegandt, Semisimple classes and $H$-relations, Studia Sci. Math. Hungar. 13 (1978), 181-185.

10072, 276 Street Department of Algebra and Geometry Mathematical Institute

WHONNOCK, B.C., VOM ISO TARTU STATE UNIVERSITY

HUNGARIAN ACADEMY OF SCIENCES

CANADA

TARTU, ESTONIA, SOVIET UNION

P.O. Box 127

H-1364 BUDAPEST 